\title{
Online Filtering for Radar Detection of Meteors
}

\author{
Eric V. C. Leite*1, Gustavo O. Alves ${ }^{1}$, José M. Seixas ${ }^{1}$, Fernando Marroquim ${ }^{2}$, \\ Cristina S. Vianna ${ }^{2}$, Helio Takai ${ }^{3}$ \\ ${ }^{1}$ Signal Processing Laboratory, COPPE/Poli, Federal University of Rio de Janeiro, Brazil. \\ 2 Physics Institute, Federal University of Rio de Janeiro, Brazil. \\ ${ }^{3}$ Brookhaven National Laboratory, USA. \\ E-mail: carvalho@lps.ufrj.br, gustavoalvesalps.ufrj.br, \\ seixas@lps.ufrj.br, marroqui@if.ufrj.br, cris_schoch@if.ufrj.br, \\ takai@bnl.gov
}

This work addresses the development of algorithms for online automatic detection of meteors using radar technique. In time-domain, the optimal filtering technique is applied. In frequencydomain, two possibilities are being studied using Short-time Fast Fourier Transform: narrowband demodulation and cumulative power spectrum analysis. The filter detecting system using the power spectrum analysis achieves $94.2 \%$ efficiency for a false alarm rate of $6.7 \%$ over 1,500 seconds of data, which could avoid to record 3.45 GB of noise every 24 hours.

13th International Workshop on Advanced Computing and Analysis Techniques in Physics Research February 22-27, 2010

Jaipur, India

${ }^{*}$ Speaker. 


\section{Introduction}

In the solar system, debris whose mass ranges from a few micrograms to kilograms are called meteoroids. By penetrating into the atmosphere, a meteoroid gives rise to a meteor, which vaporizes by sputtering, causing a bright and ionized trail that is able to scatter forward Very High Frequency (VHF) electromagnetic waves. This fact inspired the Radio Meteor Scatter technique (RMS) [1], which aims at detecting meteors using passive radar [2]. As a result of efficient detection, meteor trails parameters like diffusion and length are analyzed, which can provide useful information for studies of the ozone layer from 80 to $120 \mathrm{~km}$ above sea level and other applications [3].

Figure 1(a) shows the principle of RMS detection. Analog TV stations, which are constantly switched on and broadcasting VHF radio waves, are used as transmitters of opportunity in order to build a passive bistatic radar system [2]. The receiver station is positioned far away from the transmitter, sufficiently to be bellow the horizon line, so that signal cannot be directly detected since the ionosphere does not usually reflect electromagnetic waves in VHF range (30 - 300 MHz). The penetration of a meteor on Earth's atmosphere creates this ionized trail, which is able to produce the forward scattering of the radio waves and the scattered signals eventually reach the receiver station.

The RMS technique has many advantages over visual meteor detection methods: it works also during the day, regardless of weather conditions, covers large areas at low cost, is able to detect small meteors (from micrograms) and can acquire data continuously. Not only meteors trails, but also many other atmospheric phenomena can scatter VHF waves and may be detected, such as lightning and e-clouds [4].

In this work, algorithms are developed for online automatic detection of meteor signals in a dense background noise environment, aiming at reducing the storage requirements and providing an efficient way of classifying signals. In time-domain, the optimal filtering technique is applied. The model assumes that the received signal is masked by an additive noise and both signal and noise statistics are used to design a linear filter that maximizes the signal-to-noise ratio [5]. This technique is known as matched filter. The detection is performed by correlating the incoming signal with replicas of the target signal components at the receiver end. In frequency-domain, two possibilities are being studied using Short-time Fast Fourier Transform (STFT): a narrowband demodulation, which basically consists in performing demodulation in filtered data in order to obtain only the signal envelope, and cumulative power spectrum analysis. The cumulative spectral power is obtained from integrating the power spectral density function, which drastically reduces the noise effect [6].

This paper is organized as it follows. The experimental data used is described in Section 2, the online algorithms are summarized in Section 3. Results are discussed in Section 4 and, finally, conclusions and perspectives are in Section 5.

\section{Experimental Data}

The setup for experimental data acquisition is shown in Figure 1(b). It includes a doubleinverted $\mathrm{V}$ antenna for a nearly vertical detection, a computer controlled radio receiver tuned to video carrier channel $3(61.25 \mathrm{MHz})$ and a personal computer equipped with a 8-channel sound 
(a)

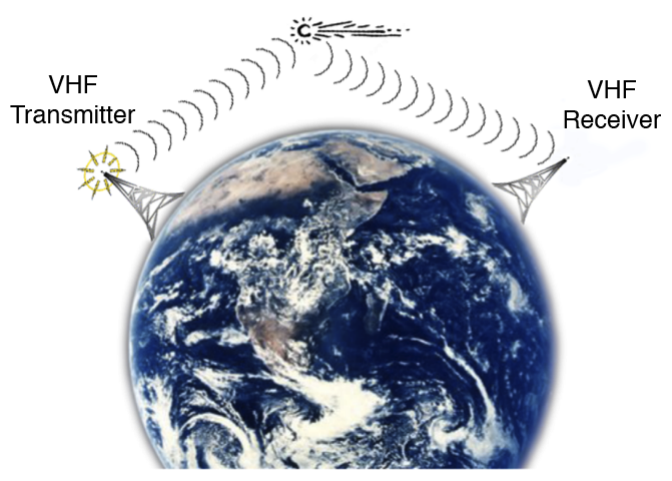

(b)

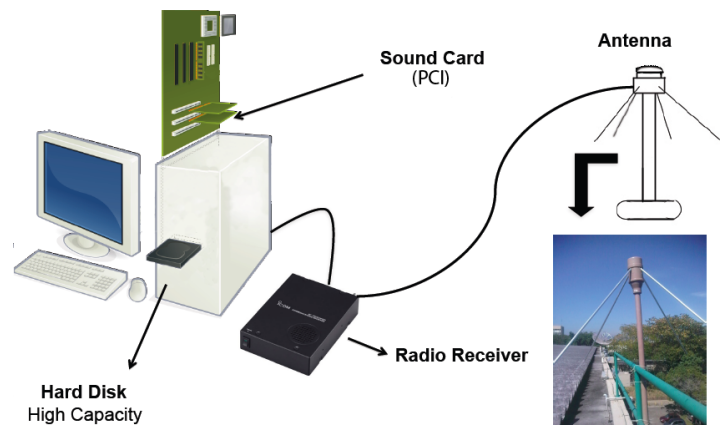

Figure 1: RMS method: (a) the detection principle and (b) the detector setup.

card, able to perform sampling rates up to $96 \mathrm{kHz}$. Considering the characteristic of continuous acquisition inherent to the radar detection technique and the generation of a significant amount of data, mainly from background noise, high-capacity hard disks are required.

What in fact reflects the VHF radio waves are the free electrons in the trails formed by meteors entering the Earth's atmosphere. But not all meteor trails have the same density of free electrons. Thus, two specific cases are defined [7]:

- Underdense trails: low density. The forward scattering is done individually by each electron. These events have a duration of few tenths of seconds and correspond to about $90 \%$ of the total data.

- Overdense trails: high density. Completely reflects the incident wave and the trail is treated as a cylindrical reflector. These events have duration from tenths to tens of seconds and form the remaining $10 \%$ of data.

Figure 2 shows a simulation of the received power for the two types of trails [8].
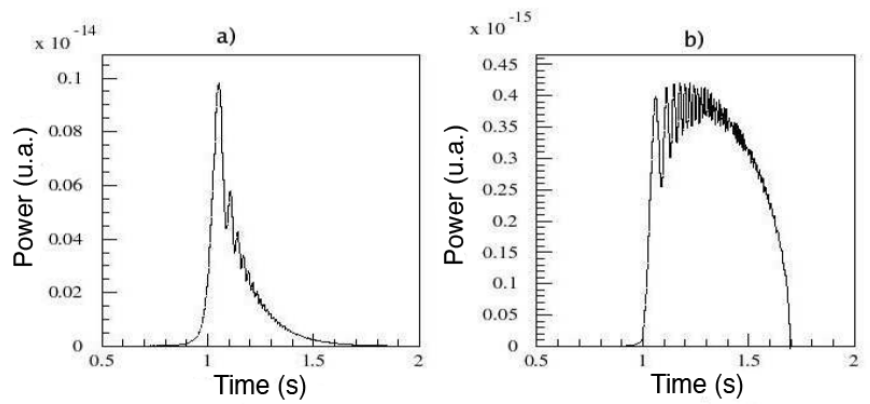

Figure 2: Simulation of (a) underdense and (b) overdense trails.

The meteor data used in this work were acquired at Custer Institute and Observatory, by the Brookhaven National Laboratory (BNL) team from June $1^{\text {st }}$ to $7^{\text {th }}, 2008$. The data were digitized using a sampling rate of $22,050 \mathrm{~Hz}$. Data from a couple of days were split into two sets: the development set, which was used for feature extraction and system design, and the test set, which aims at evaluating performance generalization. For the cumulative power spectrum algorithm, the 
development set comprised 40 data blocks, which contained 223 meteor events, and the test set was composed of 50 blocks, which contained 261 meteor events. For the matched filter, 120 data blocks were selected, which contained meteor events and noise in a equal proportion. The data were distributed equally for both development and test sets. All meteor events and noise data were selected through visual inspection.

\section{Online Algorithms}

In frequency-domain, a cumulative power spectrum analysis is applied. Information is concentrated within a narrow band of the spectrum, corresponding to the demodulated video carrier. Due to continuous acquisition, the STFT [9] is used. The signal is windowed using non-overlapping rectangular windows of 256 samples, corresponding to a time length of approximately $10 \mathrm{~ms}$, which provides a good time resolution for the detection. Blocks containing 30 seconds of acquired data are analyzed at a time by the online algorithm, corresponding to 2,584 windowed segments. For each segment, a Fast Fourier transform (FFT) is applied and the power spectral density (PSD) is estimated via periodogram [9]. Figure 3 shows the power spectrum for a data block (30s). The PSD determines the frequency bin in which information is maximum. The power peak value for each data window is then stored.

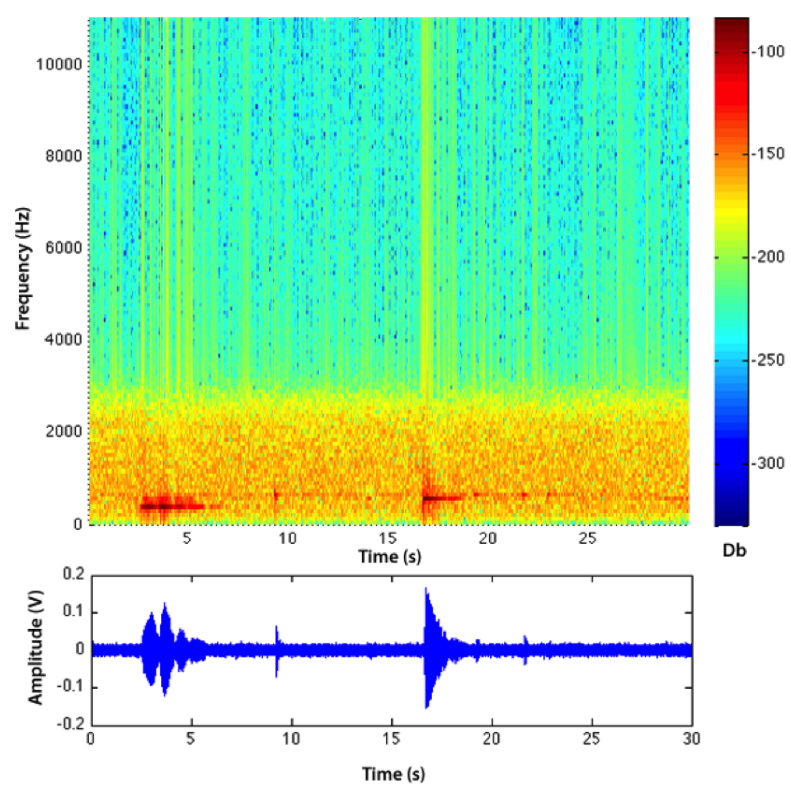

Figure 3: Spectrogram of a data block .

Figure 4(a) shows a typical power peak distribution for an underdense trail. This curve is then accumulated over all time windows, producing the curve shown in Figure 4(b). The accumulating process produces a curve that exhibits small fluctuation and monotonically increases. The slope in the cumulative power is due to the background noise and it is estimated through a linear fit, which is then subtracted from the signal. The resulting accumulated curve is shown in Figure 4(b), in the 
detail. Fluctuations in the slope threshold values are used to define the starting and ending samples for triggering.

a)

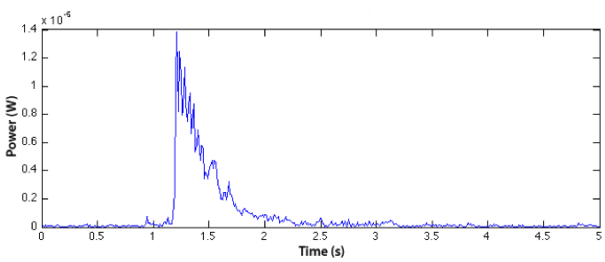

b)

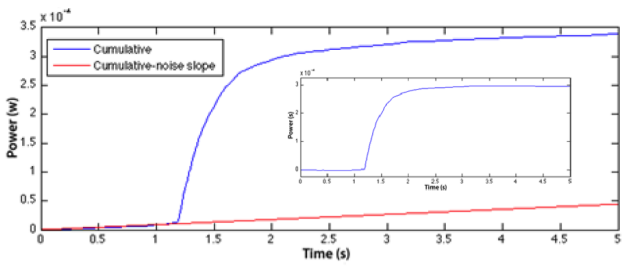

Figure 4: Underdense trail: (a) received power peaks, (b) cumulative power and noise slope (the detail corresponds to the cumulative power after subtracting the noise slope).

Two other techniques for online meteor signal detection are also being studied: a narrowband demodulation using STFT and the matched filter. In frequency-domain, the narrowband demodulation consists in performing demodulation in filtered data in order to obtain only the signal envelope [10]. Demodulation is attractive, as phase delays are produced by the different paths the traveling wave finds between the transmitting and receiving antennas, as shown in Figure 2.

Figure 5 shows the block diagram of such demodulation. Acquired time signals are filtered by a bandpass filter, typically centered at $100 \mathrm{kHz}$, the frequency band of meteor signals. In sequence, signal is squared as in a traditional demodulation scheme and a TPSW (Two Pass Split Window) algorithm is used to estimate and remove the mean of the background noise [10]. Resampling is then performed to reach the band frequency of interest that ranges from DC to $1 \mathrm{kHz}$. Finally, FFT is applied to short lengths of time, which are selected from a moving Hanning window [9] (approximated length: $500 \mathrm{~ms}$ ).

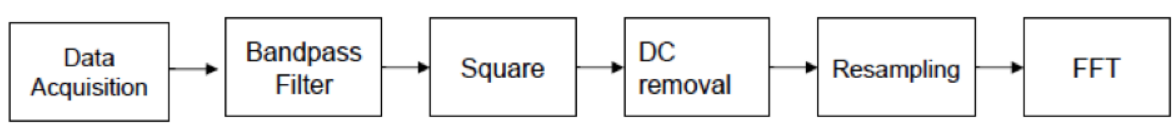

Figure 5: Block diagram for narrowband demodulation

The detection by a matched filter is optimal in the sense of the signal-to-noise ratio when the additive noise is white [11]. In practice, for Gaussian signal processes, detection is performed by correlating the incoming signal with replicas of the target signal components in the receiver end (see Figure 6). For signal detection, the determination of the detection threshold depends on the detection efficiency and the amount of the false alarm probability that can be handled. In order to reduce complexity, the matched filter design will be based on a crude approximation, which considers the stochastic meteor signal represented by it's process mean.

\section{Results}

The cumulative power spectrum algorithm detected 17 fake events over 1,500 seconds of data, which means approximately 1 fake event per each 100 seconds. Therefore, from this test sample, the algorithm avoided 220 fake events to be recorded (144 MB less per hour). In a full day, the online filter would avoid 3.45 GB of noise to be recorded. 


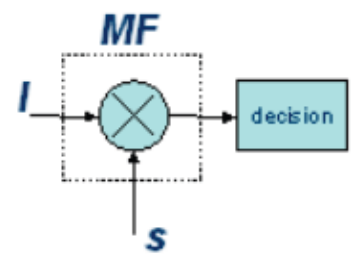

Figure 6: The matched filter (MF) detector block diagram.

On the detector setup, the computer runs both signal acquisition and processing. The signal detection requires a buffer to store a block of data (30 seconds) and processes the current data block. The time required for processing each block of data was found to be approximately 0.7 seconds, which means that no parallel processing is required for online implementation.

For the matched filter, a noise analysis has been made. Figure 7(a) shows the corresponding Gaussian fit to the distribution of the noise samples. As a first approximation, noise can be considered Gaussian. The noise covariance matrix is shown in Figure 7(b) exhibiting small crosstalks. This points out a deviation from a fully white noise process. Thus, a whitening pre-processing block had to be added [11].

(a)

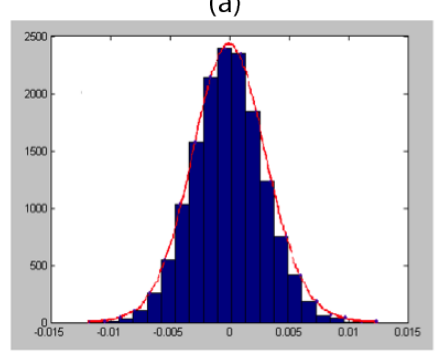

(b)

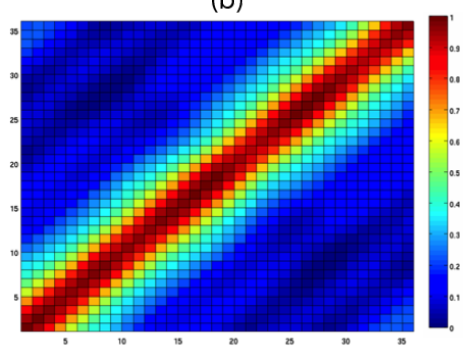

Figure 7: Noise analysis: (a) distribution and (b) covariance matrix.

Figure 8 shows the good performance of the whitening filter for both development and test set considering noise samples, exhibiting a practically diagonal covariance matrix for the whitened noise.

(a)

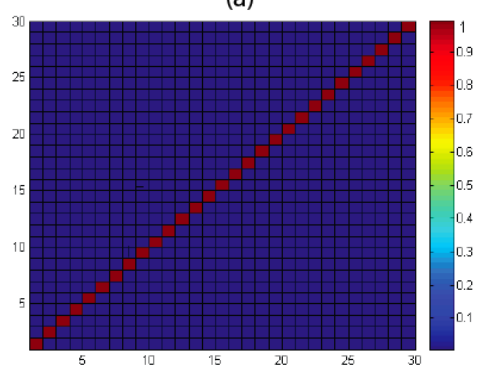

(b)

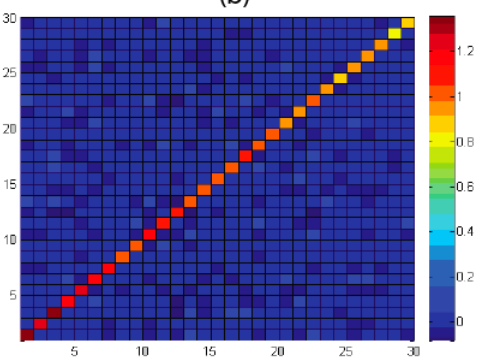

Figure 8: Covariance of the whitened noise for both (a) development and (b) test set 


\section{Conclusions and Prospects}

Using the RMS method, three algorithms for online automatic detection of meteors were proposed. The cumulative spectral analysis provided an efficient online meteor signal detection for continuous data storage with a fake rate of $6.7 \%$. Detection efficiency may be further increased by applying a whitening process to the incoming data and by using the accumulated received power at full width at half maximum, instead of using peak values.

The matched filter and the narrowband demodulation techniques are ongoing activities. More events are being selected for filter development. Preliminary results of the narrowband demodulation algorithm are being analyzed.

\section{Acknowledgments}

The authors would like to thank the BNL team for providing the experimental data and the support of the following Brazilian Agencies: CAPES, CNPq and FAPERJ.

\section{References}

[1] http://www.imo.net/radio. Acess on 2010-04-29.

[2] Willis, N.C., 'Bistatic Radar', chapter 23 in Radar Handbook, third edition, (M.I. Skolnik ed.), McGraw-Hill, 2008.

[3] Groves G.V. A theory for determining upper-atmosphere winds from radio observations on meteor trails, (1959) Journal of Atmospheric and Terrestrial Physics, 16 (3-4), pp. 344-356. McGraw-Hill, 2008.

[4] E.R. Williams, V. Mazur and S.G. Geotis, Lightning investigations with radar. In Radar in Meteorology, American Meteorological Society, Boston, Mass (1990), pp. 143-150.

[5] H.L.Van Trees. Detection, Estimation, and Modulation Theory, Part I, Wiley, 2001.

[6] R. D. Peters, A new tool for seismology-the Cumulative Spectral Power,(2007). http://arxiv.org/abs/0705.1100. Acess on 2010-04-29.

[7] J. M. Wislez, Forward scattering of radio waves of meteor trails, Proceedings of the International Meteor Conference, 83-98, September 1995.

[8] Vianna, C. S., Meteor Detection Using Forward Scattering of VHF Radio Waves, Master Dissertation. Physics Institute, Federal University of Rio de Janeiro. (In portuguese).

[9] Oppenheim, A.V., and R.W. Schafer, Discrete-Time Signal Processing, Prentice-Hall, 1989, pp.730-742.

[10] Nielsen, R. O. Sonar Signal Processing, Artech House Inc, Nortwood, MA, (1991).

[11] K.S.Shamugan, A.M. Breipohl. Random Signals - detection, estimation and data analysis,Wiley,1998. 